\title{
Chest computed tomography using iterative reconstruction vs filtered back projection (Part 1): evaluation of image noise reduction in 32 patients
}

\author{
François Pontana • Julien Pagniez • Thomas Flohr • \\ Jean-Baptiste Faivre • Alain Duhamel • Jacques Remy • \\ Martine Remy-Jardin
}

Received: 1 July 2010 /Revised: 13 September 2010 /Accepted: 8 October 2010 / Published online: 5 November 2010

(C) European Society of Radiology 2010

\begin{abstract}
Objective To assess noise reduction achievable with an iterative reconstruction algorithm.

Methods 32 consecutive chest CT angiograms were reconstructed with regular filtered back projection (FBP) (Group 1) and an iterative reconstruction technique (IRIS) with 3 (Group 2a) and 5 (Group 2b) iterations.

Results Objective image noise was significantly reduced in Group 2a and Group 2b compared with FBP $(p<0.0001)$. There was a significant reduction in the level of subjective image noise in Group 2a compared with Group 1 images $(p<0.003)$, further reinforced on Group 2b images (Group $2 \mathrm{~b}$ vs Group 1; $<<0.0001$ ) (Group 2b vs Group 2a; $p=$ $0.0006)$. The overall image quality scores significantly
\end{abstract}

F. Pontana $\cdot$ J. Pagniez $\cdot$ J.-B. Faivre $\cdot$ J. Remy $\cdot$ M. Remy-Jardin Department of Thoracic Imaging Hospital Calmette (EA 2694), Univ Lille Nord de France,

59000 Lille, France

T. Flohr

Computed Tomography Division, Siemens HealthCare,

91301 Forchheim, Germany

A. Duhamel

Department of Medical Statistics, Univ Lille Nord de France, 59000 Lille, France

\section{Remy-Jardin ( $\varangle)$}

Department of Thoracic Imaging, Hospital Calmette,

Boulevard Jules Leclercq,

59037 Lille cedex, France

e-mail: martine.remy@chru-lille.fr improved on Group 2a images compared with Group 1 images $(p=0.0081)$ and on Group $2 \mathrm{~b}$ images compared with Group 2a images $(p<0.0001)$. Comparative analysis of individual CT features of mild lung infiltration showed improved conspicuity of ground glass attenuation $(p<$ $0.0001)$, ill-defined micronodules $(p=0.0351)$ and emphysematous lesions $(p<0.0001)$ on Group 2a images, further improved on Group $2 \mathrm{~b}$ images for ground glass attenuation $(p<0.0001)$, and emphysematous lesions $(p=0.0087)$.

Conclusion Compared with regular FBP, iterative reconstructions enable significant reduction of image noise without loss of diagnostic information, thus having the potential to decrease radiation dose during chest CT examinations.

Keywords Radiation dose $\cdot \mathrm{CT} \cdot$ Chest $\cdot$ Iterative reconstruction · Image quality

\section{Introduction}

Since its introduction, CT has become the major imaging modality for investigating chest disorders, a position reinforced over years by the various technological advances successively introduced in clinical practice. Among them, multidetector CT (MDCT) has definitely influenced the evaluation of airways and lung diseases, allowing examination of the entire thorax without gaps, providing simultaneous availability of several series of images from a single data set with a dramatic reduction in breathing 
artefacts inherent to the high speed of data acquisitions [13]. This technology has also become the gold standard for diagnosing pulmonary vascular diseases, combining high spatial resolution and an optimal enhancement of thoracic circulations, exemplified in the diagnosis of acute pulmonary embolism [4]. More recently, it has become possible to integrate cardiac and coronary artery imaging into chest $\mathrm{CT}$ examinations, a concept applicable to numerous thoracic diseases [5]. The consequence of such developments is the substantial increase in the radiation dose delivered to patients, individually but also collectively [6], and the subsequent risks, even small, of cancer induction by lowlevel radiation [7, 8]. Facing the challenge of providing diagnostic image quality at the lowest radiation dose, the radiological community has modified chest $\mathrm{CT}$ protocols at the pace of new technology implementations aiming to reduce radiation exposure, such as anatomical tube current modulation, ECG-controlled tube current modulation or dynamically adjustable pre-patient collimation of the X-ray beam in the $\mathrm{z}$-axis direction $[9,10]$. Simple dose saving behaviours have also been introduced in the radiologists' daily practice, such as the adjustment of the CT parameters selected at the console, further optimized when individually-adapted, which can be associated with automatic tube current modulation systems [11, 12]. More recently, another option to save dose is the use of iterative reconstruction techniques [13].This two-part study was initiated to evaluate the performance of a recently introduced iterative reconstruction technique (Iterative Reconstruction in Image Space IRIS) [14]. In part 1 of the study, we evaluated image noise reduction achievable on routine weight-based chest CT examinations; in part 2, we implemented this technique for saving dose in a nonselected adult population.

\section{Materials and methods}

\section{Study population}

In order to evaluate the level of noise reduction and overall image quality with an iterative reconstruction algorithm, chest CT examinations of 32 consecutive patients referred for a standard CT angiographic examination were included, each data set being systematically reconstructed with regular filtered back-projection (FBP), which is the standard approach for medical CT today, and using an iterative reconstruction technique (IRIS; Siemens Healthcare, Forchheim, Germany). The sample size necessary for this evaluation was estimated on the basis of the following parameters: (a) the main purpose of this study was to compare the image noise level between two techniques, namely the standard FBP reconstructions and the iterative reconstructions obtained after 3 iterations; (b) on the standard FBP reconstructions, the mean image noise measured at the level of the trachea on mediastinal images had been previously estimated at $22.6 \pm 6$ Hounsfield units (HU) [15]; (c) our hypothesis being a noise reduction of $20 \%$ with the iterative reconstruction technique using 3 iterations, and assuming a correlation value of 0.2 between two measurements of noise with the two reconstruction techniques, an estimated number of 32 patients was needed for $90 \%$ power with significance at $5 \%$.

The study protocol was approved by our institutional Ethics Committee; no patient's informed consent was required to generate the two series of reconstructions; the retrospective analysis of data was possible with waiver of patients' informed consent. The study group was composed of 19 males and 13 females with a mean $( \pm \mathrm{SD})$ age of 58 $( \pm 18)$ years (range: $23-91)$, referred for various respiratory disorders, including bronchial and/or lung parenchymal $(n=$ 19), pulmonary vascular $(n=8)$, pleural $(n=3)$ and mediastinal $(n=2)$ diseases. The mean $( \pm \mathrm{SD})$ weight and the mean $( \pm \mathrm{SD})$ body mass index of our study group were 66.1 $( \pm 12.5) \mathrm{kg}$ (range: $50-100)$ and $23.5( \pm 5.5) \mathrm{kg} / \mathrm{m} 2$ (range: 17-42).

\section{CT protocol}

$\mathrm{CT}$ angiograms were obtained on a dual-source 128-slice MDCT system (Somatom Definition Flash, Siemens, Germany), using two tubes with single energy in a non electrocardiogram (ECG)-gated mode, with the following parameters: collimation: $64 \times 2 \times 0.6 \mathrm{~mm}$ with $\mathrm{z}$-flying focal spot; weight-adapted selection of the kilovoltage for both tubes (ranging between 100 and $120 \mathrm{kV}$ ) with adapted milliamperage setting (ranging between 90 and 100 effective mAs); 4D dose modulation (Care Dose 4D); pitch: 3; rotation time: $0.28 \mathrm{~s}$. The injection protocol consisted of the administration of $120 \mathrm{ml}$ of a contrast agent with $350 \mathrm{mg}$ of iodine per milliliter (Xenetix 350, Guerbet) at a flow rate of $4 \mathrm{ml} / \mathrm{s}$. The examination was initiated by bolus tracking within the ascending aorta with a threshold of $150 \mathrm{HU}$ to trigger data acquisition. The mean $( \pm \mathrm{SD}$ ) dose-length-product of the $\mathrm{CT}$ angiograms was 125.5 $( \pm 53.5) \mathrm{mGy} . \mathrm{cm}$, ranging from 48 to $263 \mathrm{mGy} . \mathrm{cm}$. The average effective dose was retrospectively calculated by multiplying the DLP value by a conversion factor of 0.017 [16], leading to a mean value of $2.13( \pm 0.9) \mathrm{mSv}$ (range: $0.81-4.47)$

Image reconstruction

While increased spatial resolution is directly correlated with increased image noise in standard filtered back-projection reconstructions as they are used in CT practice today, 
iterative reconstruction approaches to a certain extent allow decoupling of spatial resolution and image noise [13]. In an iterative reconstruction, a correction loop is introduced. Once an image has been reconstructed from the measured projections, a ray-tracing in the image is performed to calculate new projections that exactly represent the reconstructed image. This step, called reprojection, simulates the CT measurement process with the image as measurement object. The deviation between measured and calculated projections is used to derive correction projections, reconstruct a correction image and update the original image. This loop is continued until the deviation between measured and calculated projections is smaller than a predefined limit. Each time the original image is updated, non-linear image processing algorithms are used to enhance spatial resolution at higher object contrasts and to reduce image noise in low contrast areas. This "regularization" step is essential for the noise reduction properties of an iterative reconstruction, whereas the repeated calculation of correction projections removes image artifacts introduced by the approximative nature of the filtered backprojection reconstruction. Regularization can as well be applied to the image data in an iterative loop without reprojection and calculation of correction projections [14]. In the "IRIS" approach, an iterative series of 3-dimensional non-linear image processing steps, corresponding to the regularization in a standard iterative reconstruction, is performed after reconstruction of an initial high resolution image. This high resolution image contains all measured information that is otherwise partially suppressed in a standard CTreconstruction to obtain acceptable image noise levels. The non-linear image processing steps are locally adapted, according to the local image noise and image structure. They aim at maintaining or even enhancing spatial resolution at higher object contrasts, while reducing image noise in low contrast areas without degrading the image texture.

From each data set, 2 series of images (i.e., 1-mm thick images) were systematically generated on a prototype workstation: (a) lung and mediastinal images reconstructed with a standard FBP algorithm (Group 1) using a high spatial resolution kernel (B50) and a soft tissue kernel (B20), respectively; (b) lung and mediastinal images reconstructed with an iterative reconstruction technique (IRIS algorithm; Siemens) (Group 2) using a high spatial resolution iterative kernel (I50) and a soft tissue iterative kernel (I20), respectively. The iterative reconstruction kernels I50f and I20f are designed to closely match the spatial resolution (in terms of the $50 \%$ and $10 \%$ value of the modulation transfer function for high contrast objects) of the corresponding filtered back-projection kernels B50f and B20f, respectively. The assessment of the IRIS technique was made on 2 levels of iterations, including reconstruc- tions using 3 iterations (Group 2a) and reconstructions using 5 iterations (Group 2b). All images were viewed at standard mediastinal (window width, $400 \mathrm{HU}$, window center, $40 \mathrm{HU}$ ) and lung parenchymal (window width, 1,600 HU; window center, -600 HU) window settings.

\section{CT parameters evaluated}

\section{Image noise}

The objective assessment of noise on Group 1, Group $2 \mathrm{a}$ and Group $2 \mathrm{~b}$ images was obtained by measuring the standard deviation of pixel values in homogeneous regions-of-interest on mediastinal (B20 and I20) images (at two anatomical levels: the tracheal lumen above the aortic arch; the descending aorta at the level of the ventricular cavities) and on lung (B50 and I50) images (at one anatomical level: the tracheal lumen above the aortic arch) using the following methodology. On standard window settings, a circular region of interest (ROI) was positioned within the tracheal lumen or the aorta. The mean density value inside the ROI was chosen as the new window centre whereas the window width was arbitrarily defined between $100 \mathrm{HU}$ and $200 \mathrm{HU}$, thus enabling the readers to confirm the homogeneity of noise within the tracheal or aortic lumina. On images viewed with the newly defined window settings, a circular ROI was again drawn within the trachea or the aorta to assess noise objectively. Care was taken to avoid superimposition of the ROI on the inner portion of the tracheal or aortic wall. The standard deviation of the measured density in HU defined the objective noise.

The visual perception of noise, defined by the grainy appearance of the CT images, was evaluated on mediastinal and lung images of Group 1, Group 2a and Group 2b images viewed with standard window settings. On each series of mediastinal and lung images, the image noise was rated as minimal (score 1) or moderate (score 2), by comparison with reference images (score 1 and 2 noise levels did not alter the identification of normal and/or abnormal structures), or important (score 3) when the image noise altered the identification of normal and/or abnormal structures.

\section{Overall image quality}

The overall image quality of lung and mediastinal images of each group of reconstructions was rated using a threepoint scale. Reconstructions with distinct anatomic detail, no noise or minimal image noise were individually rated with a score 1 (excellent image quality). Reconstructions with clear anatomic detail and moderate increase in noise 
that did not affect their diagnostic value were individually rated with a score of 2 (good image quality without impairment of diagnostic accuracy), whereas examinations with a marked increase in noise altering their diagnostic value were individually rated with a score of 3 (non diagnostic examinations). When lung and mediastinal images of a given examination had different scores, the worst image quality was systematically considered to rate the overall image quality of the examination.

\section{Lesion conspicuity in group 2}

In order to evaluate the potential impact of iterative reconstructions on lesion conspicuity, we selected 3 lung images per patient on Group 1 reconstructions, each of them showing at least one of the following elementary lesions: ground-glass opacities, ill-defined micronodules, well-defined micronodules, septal lines, nonseptal lines, focal areas of hypoattenuation with (i.e., lung cysts) or without (i.e., centrilobular emphysematous lesions) definable walls. Lung images at the corresponding anatomical levels on Groups $2 \mathrm{a}$ and $2 \mathrm{~b}$ reconstructions were selected in order to visually assess the presence of the elementary lesion(s) and to grade their conspicuity as equivalent, superior or inferior to that on Group 1 images. An additional evaluation of lesion conspicuity was made between Group 2a and Group $2 b$ to determine a potential difference between the two series of images.

\section{Conditions of image analysis}

The image quality assessment was performed by 2 subspecialty thoracic radiologists (**BLINDED**) with 2 and 6 years experience in $\mathrm{CT}$, respectively. They evaluated the CT parameters by consensus on a workstation. The FBP reconstructions were performed using the CT system's built in reconstruction computer. For the iterative reconstructions, raw data were transferred to an offline PC provided by the vendor. The reconstructed images were dicomized and sent back to a clinical workstation for viewing and measurement of image noise.

\section{Statistical analysis}

Results were expressed as means and standard deviations for continuous variables, frequencies and percentages for categorical variables. For continuous variables, comparisons between reconstruction techniques were performed using a two-way analysis of variance (ANOVA). We used a linear mixed model with the patients considered as a random effect and the reconstruction technique as a fixed effect. For categorical parameters, comparisons were performed using the McNemar chi-square test. All pairwise comparisons were performed with a Bonferroni correction. The statistical significance was defined as $p<0.05$. The statistical analyses were performed using the SAS software (SAS Institute Inc Cary, NC 25513,USA).

\section{Results}

Evaluation of image noise

\section{Objective image noise}

There was no statistically significant difference between the mean surfaces of the circular ROIs used for objective noise assessment $(p>0.05)$.

In Table 1,s the mean values of objective noise measurements on mediastinal and lung images reconstructed with FBP (Group 1), 3 iterations (Group 2a) and 5 iterations (Group 2b) are compared. There was a significant reduction in the objective noise in Group 2a compared with Group 1, with a mean noise reduction of $34.3 \%$ on lung images and a mean reduction of $31.5 \%$ at the level of the aorta and $37.2 \%$ at the level of the trachea on mediastinal images (ANOVA with Bonferroni correction; $p<0.0001$ ).

The objective noise was further reduced on Group $2 \mathrm{~b}$ images: (a) compared with Group 2a, the mean noise reduction was $14.2 \%$ at the level of the aorta and $14.5 \%$ at the level of the trachea on mediastinal images and 15.2\% on lung images (ANOVA with Bonferroni correction; $p<$ 0.0001 ); (b) leading to an overall noise reduction of $49.5 \%$ on lung images and a noise reduction of $45.7 \%$ at the level of the aorta and $51.7 \%$ at the level of the trachea on mediastinal images when compared with Group 1 images (ANOVA with Bonferroni correction; $p<0.0001$ ).

\section{Subjective image noise}

The distributions of subjective image quality scores in Group 1, 2a and $2 \mathrm{~b}$ are summarized in Table 1 . There was a significant reduction in the level of subjective image noise on lung and mediastinal images of Group 2a compared with Group 1 images $(p<0.003)$, further reinforced on Group 2b images (Group 2b vs Group 1; $p<0.0001$ ) (Group 2b vs Group 2a; $p=0.0006$ ) where image noise was rated as minimal in $78 \%$ of mediastinal images and $100 \%$ of lung images (McNemar chi-square test with Bonferroni correction).

Evaluation of the overall image quality

Table 1 summarizes the distributions of the overall image quality scores, significantly improved on Group 2a images compared with Group 1 images $(p=0.0081)$ and on Group 
Table 1 Comparison of image quality between iterative reconstructions and filtered back projections

\begin{tabular}{|c|c|c|c|}
\hline & $\begin{array}{l}\text { Group } 1 \\
\text { Images reconstructed with } \\
\text { FBP (references images) }\end{array}$ & $\begin{array}{l}\text { Group } 2 \mathrm{a} \\
\text { Images reconstructed } \\
\text { with } 3 \text { iterations }\end{array}$ & $\begin{array}{l}\text { Group } 2 \mathrm{~b} \\
\text { Images reconstructed } \\
\text { with } 5 \text { iterations }\end{array}$ \\
\hline \multicolumn{4}{|l|}{ Comparison of objective noise } \\
\hline $\begin{array}{l}\text { Objective noise at the level of the trachea on mediastinal } \\
\text { images mean (SD), HU }\end{array}$ & $22.26( \pm 10.10)$ & $13.98( \pm 7.86) *$ & $10.75( \pm 6.67) * \S$ \\
\hline $\begin{array}{l}\text { Objective noise at the level of the aorta on mediastinal } \\
\text { images mean (SD),HU }\end{array}$ & $48.00( \pm 13.99)$ & $32.87( \pm 10.66)^{*}$ & $26.08( \pm 9.13)^{*} \S$ \\
\hline $\begin{array}{l}\text { Objective noise at the level of the trachea on lung images } \\
\text { mean (SD),HU }\end{array}$ & $56.47( \pm 16.87)$ & $37.08( \pm 12.19)^{*}$ & $28.53( \pm 10.06)^{*} \S$ \\
\hline \multicolumn{4}{|l|}{ Comparison of subjective noise } \\
\hline \multirow[t]{3}{*}{ Subjective image noise on mediastinal images } & -score 1: 0 & -score 1: $11(34 \%)$ & -score 1: $25(78 \%)$ \\
\hline & -score 2: $32(100 \%)$ & -score 2: $21(66 \%)$ & -score $2: 7(22 \%)$ \\
\hline & -score 3: 0 & -score 3: 0 & -score 3: 0 \\
\hline \multirow[t]{3}{*}{ Subjective image noise on lung images } & -score 1: 0 & -score 1: $18(56 \%)$ & -score 1: $32(100 \%)$ \\
\hline & -score 2: $32(100 \%)$ & -score 2: $14(44 \%)$ & -score 2: 0 \\
\hline & -score 3: 0 & -score 3: 0 & - score $3: 0$ \\
\hline \multicolumn{4}{|l|}{ Comparison of distribution of the overall image quality scores } \\
\hline Excellent image quality (score 1) & 0 & $9(28 \%)$ & $25(78 \%)$ \\
\hline Good image quality (score 2) & $32(100 \%)$ & $23(72 \%)$ & $7(22 \%)$ \\
\hline Nondiagnostic image quality (score 3 ) & 0 & 0 & 0 \\
\hline
\end{tabular}

FBP filtered backprojection; $H U$ Hounsfield Unit

NB: The statistical comparisons were obtained as follows. The two-by-two comparisons of objective noise were made between groups using a two-way analysis of variance (ANOVA) with Bonferroni correction. * refers to statistically significant differences with Group $1(*=p<0.0001)$; $\S$ refers to statistically significant differences with Groups $2 \mathrm{a}(\xi=p<0.0001)$. The pairwise comparisons of subjective noise were obtained using $\mathrm{McNemar}$ chi-square test with a Bonferroni correction. The distribution of the overall image quality scores was compared using the McNemar test with Bonferroni correction.

2b images compared with Group 2a images $(p<0.0001)$ (McNemar chi-square test with Bonferroni correction). Figure 1 illustrates the reduction in image noise obtained with iterative reconstructions in an obese patient.

\section{Lesion conspicuity}

A total of 96 lung images with at least one elementary lesion of mild lung infiltration were selected in Group 1 at
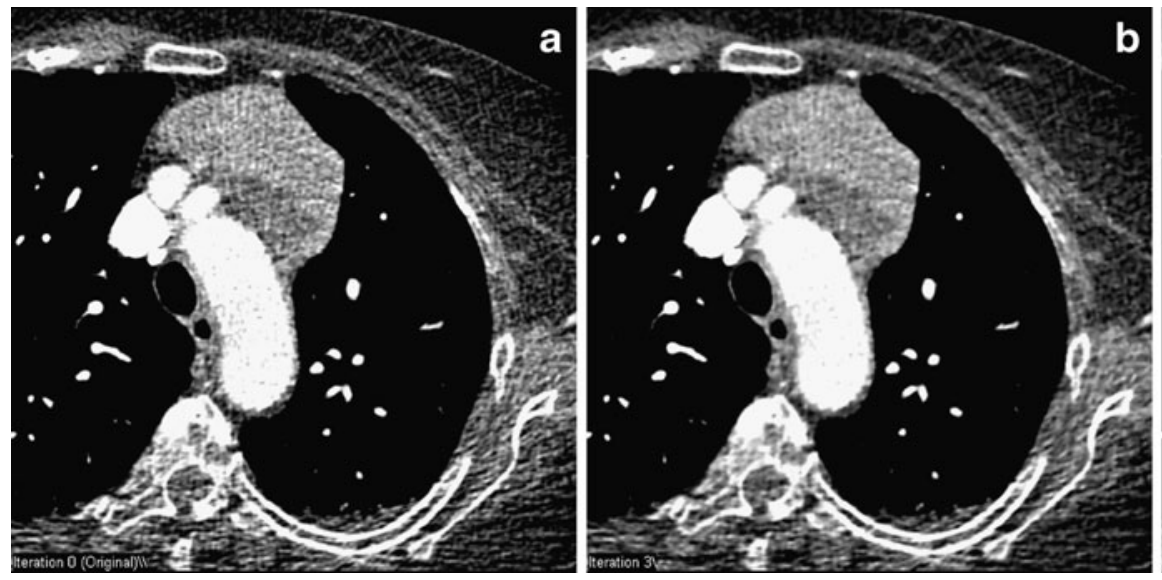

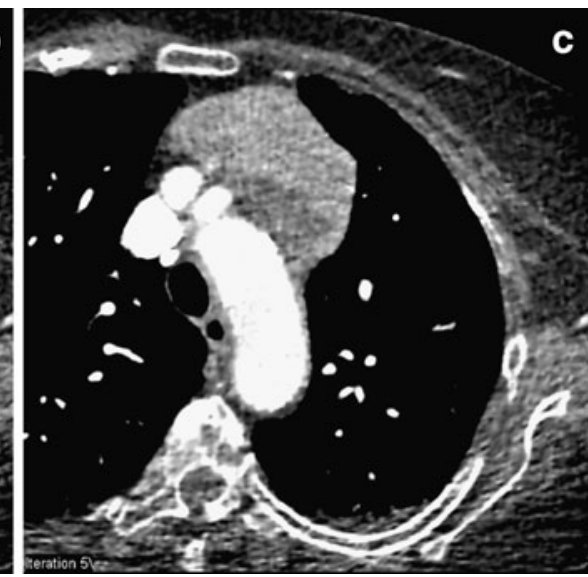

Fig. 1 CT angiographic examination in a 65 year-old obese woman (BMI: $41.4 \mathrm{~kg} / \mathrm{m} 2)$ referred for mediastinal mass $(140 \mathrm{kV} ; 120$ eff mAs; DLP: 263 mGy.cm), illustrating the reduction in objective image noise with 3 and 5 iterations. a: FBP-reconstructed mediastinal image at the level of the aortic arch. Objective noise measured at the level of the trachea: $37.6 \mathrm{HU}$. Subjective noise: score $2 \mathbf{b}$ : Same anatomical level as that of (a), reconstructed with 3 iterations. Objective noise measured at the level of the trachea: 27HU. Subjective noise: score 2 c: Same anatomical level as that of (a), reconstructed with 5 iterations. Objective noise measured at the level of the trachea: $21.8 \mathrm{HU}$. Subjective noise: score 2 
Table 2 Comparative analysis of lesion conspicuity on lung images showing features of mild lung infiltration

\begin{tabular}{|c|c|c|c|c|c|c|c|}
\hline & \multirow{2}{*}{$\begin{array}{l}\text { Number of lung CT } \\
\text { sections showing the } \\
\text { abnormal CT features }\end{array}$} & \multicolumn{2}{|c|}{ Group 2a vs Group 1} & \multicolumn{2}{|c|}{ Group $2 \mathrm{~b}$ vs Group 1} & \multicolumn{2}{|c|}{ Group 2b vs Group 2a } \\
\hline & & Equal & Superior & Equal & Superior & Equal & Superior \\
\hline $\begin{array}{r}\text { Areas of ground } \\
\text { glass opacities }\end{array}$ & 49 & 0 & $49(100 \%) p<0.0001$ & 0 & $49(100 \%) p<0.0001$ & 13 & $36(73.5 \%) p<0.0001$ \\
\hline $\begin{array}{l}\text { Ill-defined } \\
\text { micronodules }\end{array}$ & 11 & 0 & $11(100 \%) p=0.0351$ & 0 & $11(100 \%) p=0.0351$ & 3 & $8(72.7 \%) p=0.1380$ \\
\hline Well-defined micronodules & 10 & 10 & 0 & 10 & 0 & 10 & 0 \\
\hline Septal lines & 5 & 5 & 0 & 5 & 0 & 5 & 0 \\
\hline Nonseptal lines & 29 & 29 & 0 & 29 & 0 & 29 & 0 \\
\hline Cysts & 4 & 4 & 0 & 4 & 0 & 4 & 0 \\
\hline $\begin{array}{l}\text { Emphysematous } \\
\text { lesions }\end{array}$ & 31 & 2 & $29(93.5 \%) p<0.0001$ & 2 & $29(93.5 \%) p<0.0001$ & 17 & $14(45.2 \%) p=0.0087$ \\
\hline
\end{tabular}

McNemar test with Bonferroni correction

the level of which we identified focal areas of ground glass attenuation on 49 images, ill-defined micronodules on 11 images, well-defined micronodules on 10 images, septal lines on 5 images, non septal lines on 29 images, lung cysts on 4 images and emphysematous lesions on 31 images. The individual CT features identified on Group 1 images were always identified on the corresponding Group 2a and Group $2 \mathrm{~b}$ images. Table 2 summarizes the comparative analyses of lesion conspicuity on paired lung images, showing: (a) a similar conspicuity of well-defined micronodules, septal and nonseptal lines, and cysts in Groups 1, $2 \mathrm{a}$ and $2 \mathrm{~b}$; (b) a better conspicuity of ground glass opacities (Fig. 2), ill-defined micronodules (Fig. 3) and emphysematous lesions (Fig. 4) on Group 2a images compared with Group 1 images; (c) a better conspicuity of ground glass opacities and emphysematous lesions on Group $2 b$ images compared with Group 2a images.
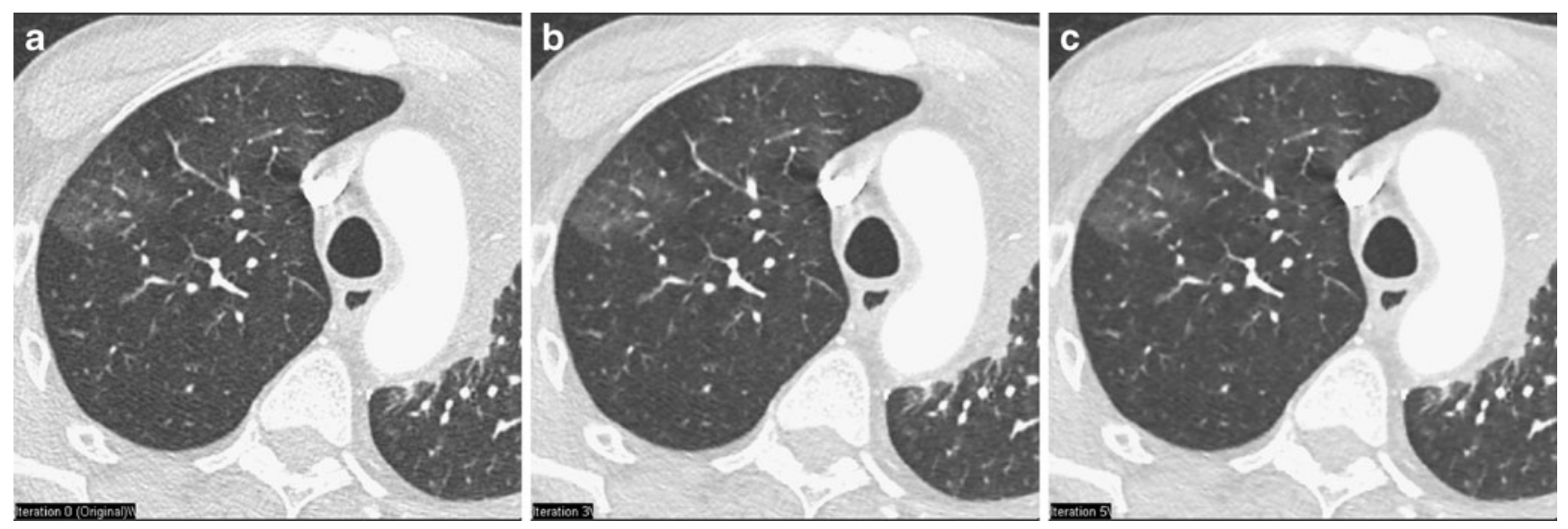

Fig. 2 CT angiographic examination in a 55 year-old man (normal BMI: $19.8 \mathrm{~kg} / \mathrm{m} 2)$ referred for lung tumor follow-up $(100 \mathrm{kV}$; 90 eff mAs; DLP: 108 mGy.cm). a: FBP-reconstructed lung image at the level of the aortic arch, showing ground glass opacities in both lungs. Objective noise measured at the level of the trachea: 42.1HU. Subjective noise: score 2 b: Same anatomical level as that of (a),

\section{Discussion}

To our knowledge, this is the first study evaluating the performance of an iterative reconstruction technique in image space (IRIS) for chest imaging. Comparing FBP and iterative reconstructions generated from the same data sets, our results demonstrate that the iterative reconstruction technique significantly reduces the level of objective image noise on standard CT examinations of the chest obtained in a non selected population of adult patients. While 3 iterations reduced the image noise by $31.5 \%$ and $37.2 \%$ on mediastinal images and by $34.3 \%$ on lung images, the greatest noise suppression was found to be obtained with 5 iterations, enabling a noise reduction ranging from $45.7 \%$ to $51.7 \%$ on mediastinal images and $49.5 \%$ on lung images compared with the FBP technique. Analysis of the distribution of subjective image noise scores showed reconstructed with 3 iterations. Objective noise measured at the level of the trachea: 26.3HU. Subjective noise: score 2. c: Same anatomical level as that of (a), reconstructed with 5 iterations. Objective noise measured at the level of the trachea: 19.3 HU. Subjective noise: score 1. Note the improved conspicuity of ground glass opacities from (a) to (c) 

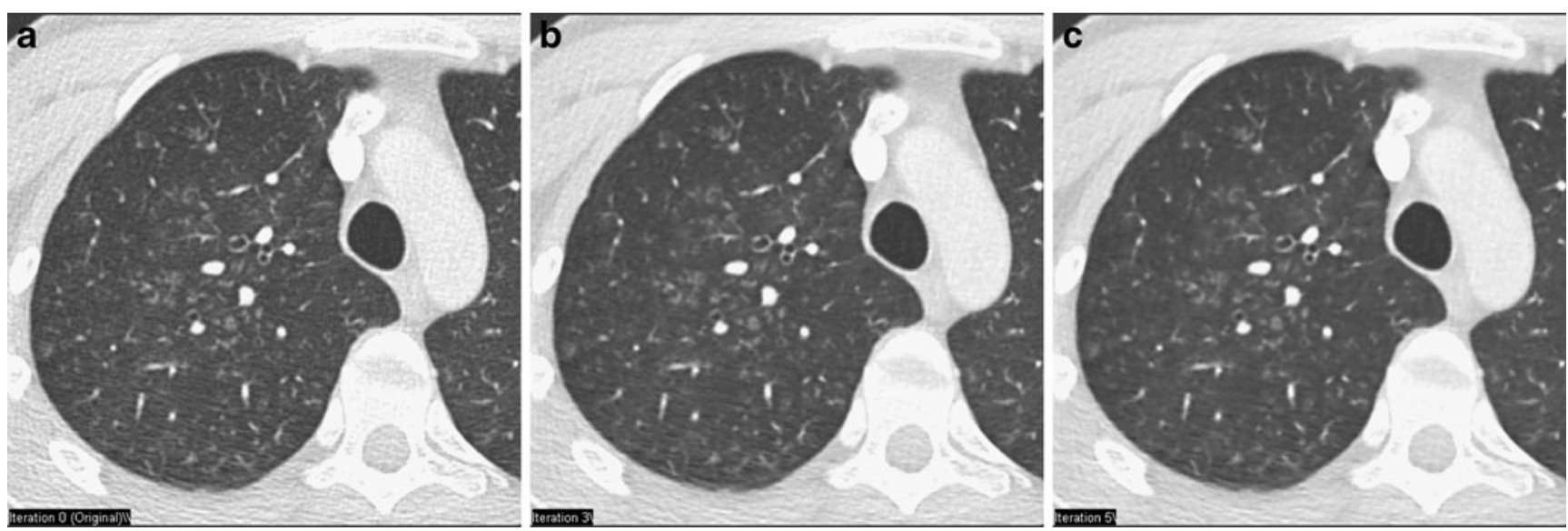

Fig. $3 \mathrm{CT}$ angiographic examination in a 30 year-old man (normal BMI: $23.55 \mathrm{~kg} / \mathrm{m} 2$ ) referred for exacerbation of chronic obstructive pulmonary disease (120 kV; 90 eff mAs; DLP: $151 \mathrm{mGy} . \mathrm{cm})$. a: FBPreconstructed lung image at the level of the right upper lobe, showing ill-defined micronodules. Objective noise measured at the level of the trachea: 45HU. Subjective noise: score 2 b: Same anatomical level as

significantly better ratings for Group 2a reconstructions compared with Group 1 reconstructions, both for lung and mediastinal images. However, the highest scores were observed in Group 2b with a minimal image noise on all lung images and in $78 \%$ of mediastinal images. The moderate image noise, always observed on our reference images, can be explained by a systematic weight-adapted selection of the kilovoltage in our daily routine. It should be pointed out that it did not alter the diagnostic value of images.

Owing to the recent introduction of other iterative reconstruction techniques, a single study reported a clinical experience of a different iterative reconstruction technique that of (a), reconstructed with 3 iterations. Objective noise measured at anatomical level as that of (a), reconstructed with 5 iterations. Objective noise measured at the level of the trachea: $20.6 \mathrm{HU}$. Subjective noise: score 1 . Note the improved conspicuity of ill-defined micronodules from (a) to (c)

(adaptive statistical iterative reconstruction-ASIR) in chest $\mathrm{CT}$ in which the authors retrospectively selected 2 groups of chest CT examinations, comparing 98 examinations reconstructed with the FBP with 54 examinations reconstructed using the ASIR [17]. Several methodological differences in the CT protocols and study designs can explain why the objective noise measured on the images reconstructed with 3 and 5 iterations in the present study is higher than that reported in the study of Prakash et al using the ASIR technique. One explanation is that the regions-ofinterest for noise measurements on the descending aorta in both studies were positioned at anatomical levels with major differences in terms of x-ray absorption, Prakash et al the level of the trachea: $28.3 \mathrm{HU}$. Subjective noise: score 2. c: Same
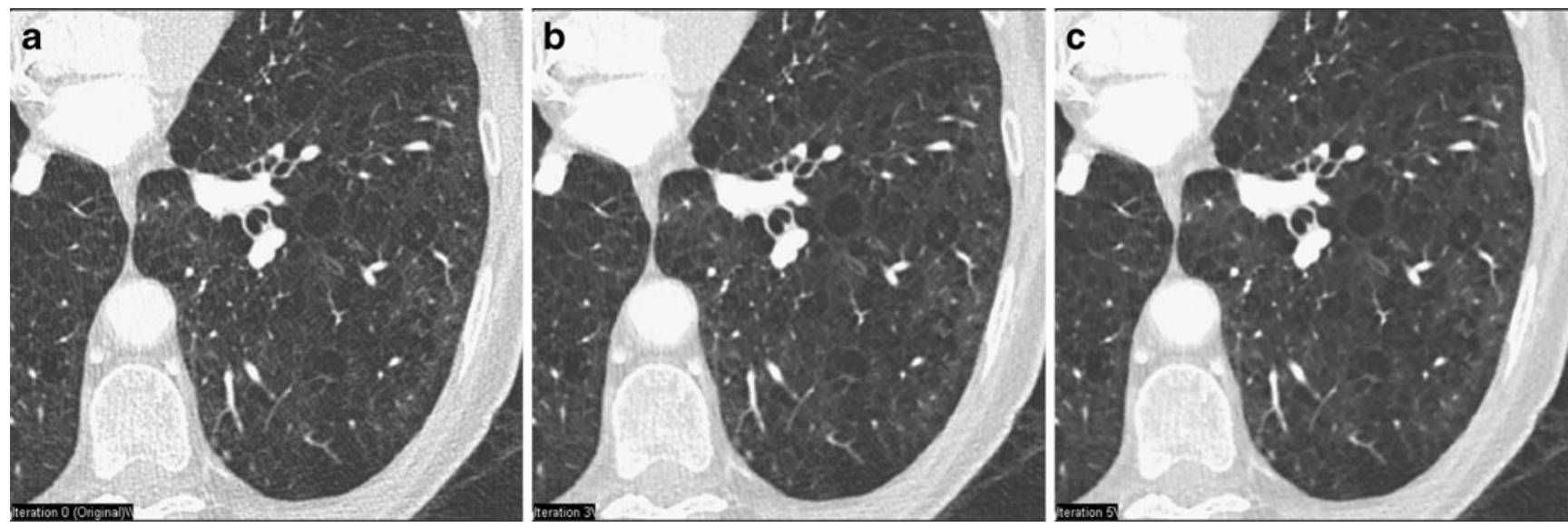

Fig. $4 \mathrm{CT}$ angiographic examination in a 59 year-old man (normal BMI: $19.3 \mathrm{~kg} / \mathrm{m} 2$ ) referred for suspicion of acute pulmonary embolism $(100 \mathrm{kV}$; 90 eff mAs; DLP: $118 \mathrm{mGy} . \mathrm{cm})$ a: FBPreconstructed lung image at the level of the left lower lobes, showing disseminated centrilobular emphysema. Objective noise measurd at the level of the trachea: $32.1 \mathrm{HU}$. Subjective noise: score $1 \mathbf{b}$ : Same anatomical level as that of (a), reconstructed with 3 iterations. Objective noise measured at the level of the trachea: $19.8 \mathrm{HU}$. Subjective noise: score 1. c: Same anatomical level as that of (a), reconstructed with 5 iterations. Objective noise measured at the level of the trachea: 14.5 HU. Subjective noise: score 1. Note the improved conspicuity of emphysematous lesions from (a) to (c) 
choosing the level of the carina while our study chose the level of the ventricular cavities. More important to consider are the major differences in the CT protocols chosen in both studies. Whereas our examinations were obtained at 100 or $120 \mathrm{kVp}$ and 90 eff mAs, the CT protocol in the patients' weight category comparable to that of our population (i.e., the $61-90 \mathrm{~kg}$ category), included $120 \mathrm{kVp}$ and a milliamperage ranging from 75 to 440 . As a result, the mean radiation dose in their study was $10 \mathrm{mSv}$ whereas our average effective dose was only $2.13 \mathrm{mSv}$, i.e., a 5-fold difference in routine clinical practice.

Our study included a specific evaluation of lesion conspicuity in our study, choosing the CT features of mild lung infiltration to test this qualitative aspect of image quality. While each elementary lesion was identified on the 3 series of images, the visual depiction of ground glass opacities, ill-defined micronodules and emphysematous lesions was improved on Group 2a compared with reference images. Further comparison between the two levels of iterative reconstructions showed greater conspicuity of ground glass opacities and emphysematous lesions on Group $2 b$ images, confirming that reduction of the objective image noise reinforces the detectability of subtle abnormalities, as also recently reported by Prakash et al with the ASIR technique [18]. Although depiction of illdefined micronodules can also be influenced by the overall image quality, their conspicuity was not found to be increased on Group 2b images, probably because of the limited number of images showing this pattern. This preliminary experience does not confirm a practical concern recently raised by $\mathrm{Xu}$ et al [19]. Considering that radiologists are used to reading images reconstructed with FBP algorithm, these authors suggested that statistical reconstruction might give an impression of somewhat reduced diagnostic value.

This study suffers from several limitations. First, the evaluation of the iterative reconstruction technique was limited to a single CT protocol, based on single energy acquisitions. This constraint was due to the fact that, at the time of initiation of this evaluation, the prototype workstation was only able to reconstruct single energy data sets. A second limitation is that we restricted our investigation to 3 and 5 iterations and did not evaluate a wider range of iterations. This resulted from vendor-recommendations to avoid potential degradations of image texture and loss of spatial resolution when using more than 5 iterations. Third, the evaluation of lesion conspicuity was limited to the comparative analysis of $\mathrm{CT}$ features of lung infiltration and did not include mediastinal structures. However, the categories of lung lesions selected for this evaluation are among the most difficult to depict and one can reasonably assume that they reflect the detectability of subtle lesions on chest CT examinations reconstructed with IRIS. Lastly, image analysis was made by consensus between two readers and did not include the assessment of inter- nor intraobserver agreement between the two radiologists enrolled in this analysis. Owing to the recent introduction of iterative reconstructions, this study design was considered most suited for a preliminary evaluation.

In conclusion, our study shows that, compared with regular FBP, iterative reconstructions enable significant reduction of image noise without loss of diagnostic information, thus having the potential to decrease radiation dose during chest CT examinations.

Acknowledgement M. Rémy-Jardin received research funding from Siemens Healthcare. J. Rémy is consultant at Siemens Healthcare. T. Flohr is an employee of Siemens Healthcare. The remaining authors (F. Pontana, J. Pagniez, J.B. Faivre, A. Duhamel) have no financial disclosures and had complete unrestricted access to study data at all stages of the study.

\section{References}

1. Schoepf UJ, Bruening RD, Hong C et al (2001) Multislice helical $\mathrm{CT}$ of focal and diffuse lung disease: comprehensive diagnosis with reconstruction of contiguous and high resolution CT sections from a single thin-collimation scan. AJR 177:179-184

2. Kelly DM, Hasegawa I, Borders R, Hatabu H, Boiselle PM (2004) High-resolution CT using MDCT: comparison of degree of motion artefact between volumetric and axial methods. AJR $182: 757-759$

3. Studler U, Gluecker T, Bongartz G, Roth J, Steinbrich W (2005) Image quality from high-resolution $\mathrm{CT}$ of the lung: comparison of axial scans and of sections reconstructed from volumetric data acquired using MDCT. AJR 185:602-607

4. Remy-Jardin M, Pistolesi M, Goodman LR, Gefter WB, Gottschalk A, Mayo JR, Sostman HD (2007) Management of suspected acute pulmonary embolism in the era of CT angiography: a statement from the Fleischner Society. Radiology 245:315329

5. Yoo SM, White CS (2008) Evaluation of chest pain, hemoptysis and dyspnea. In: Remy-Jardin M, Remy J (eds) Integrated cardiothoracic imaging. Springer-Verlag, Berlin, pp 221-238

6. Sodickson A, Baeyens PF, Andriole KP, Prevedello LM, Nawfel RD, Hanson R, Khorasani R (2009) Recurrent CT, cumulative radiation exposure and associated radiation-induced cancer risks from CT of adults. Radiology 251:175-184

7. Huang B, Wai-Ming Law M, Khong PL (2009) Whole-body PET/ CT scanning: estimation of radiation dose and cancer risk. Radiology 251:166-174

8. Cardis E, Vrijheid M, Blettner M et al (2007) The 15-country collaborative study of cancer risk among radiation workers in the nuclear industry: estimates of radiation-related cancer risks. Radiation Research 167:396-416

9. Kalender WA, Buchenau S, Kellermeier M, Langner O, van Straten M, Vollmar S, Wilharm S (2008) Technical approaches to the optimization of CT. Phys Med 24:71-79

10. Christner JA, Zavaletta VA, Eusemann CD, Walz-Flannigan AI, McCollough CH (2010) Dose reduction in helical CT: dynamically adjustable z-axis x-ray beam collimation. AJR 194:W49-55

11. Kubo T, Lin PJP, Stiller W, Takahashi M, Kauczor HU, Ohno Y, Hatabu H (2008) Radiation dose reduction in chest CT: a review. AJR 190:335-343 
12. Mayo KR, Leipsic JA (2009) Radiation dose in cardiac CT. AJR 192:646-653

13. Thibault JB, Sauer KD, Bouman CA, Hsieh J (2007) A threedimensional statistical approach to improved image quality for multislice helical CT. Med Phys 34:4526-44

14. 14-Bruder H, Raupach R, Sedlmair M, Sunnegardh J, Stierstorfer K, Flohr TG (2010) Reduction of radiation dose in CT with an FBP-based iterative reconstruction technique. (abstract), B-568. Insight into Imaging (ECR abstract book) 2010: S131

15. Tacelli N, Remy-Jardin M, Flohr T, Faivre JB, Delannoy V, Duhamel A, Remy J (2010) Dual-source chest CT angiography with high temporal resolution and high pitch modes: evaluation of image quality in 140 patients. Eur Radiol 20:1188-1196
16. 16-EC99 (1999) European guidelines on quality criteria for computed tomography. Report EURO 16262 EN. Luxembourg; 69-78

17. Prakash P, Kalra MK, Digumarthy SR, Hsieh J, Pien H, Singh S, Gilman MD, Shepard JAO (2010) Radiation dose reduction with chest computed tomography using adaptive statistical iterative reconstruction technique: initial experience. J Comput Assist Tomogr 34:40-45

18. Prakash P, Kalra MK, Ackman JB, Digumarthy SR, Hsieh J, Do S, Shepard JAO, Gilman MD (2010) Diffuse lung disease: CT of the chest with adaptive statistical iterative reconstruction technique. Radiology 256:261-269

19. Xu J, Mahesh M, Tsui BMW (2009) Is iterative reconstruction ready for MDCT? J Am Coll Radiol 6(274):276 